\title{
COSTOS COMO FACTOR CLAVE EN LA GESTIÓN DE PROYECTOS DE SOFTWARE
}

\section{COSTS AS KEY FACTOR IN THE MANAGEMENT OF SOFTWARE PROJECTS}

\author{
Esp. Jaime Yair Serrano Salazar, MSc. Maritza Del Pilar Sánchez Delgado \\ Universidad de Pamplona \\ * Centro de Investigación Aplicada y Desarrollo en Tecnologías de Información (CIADTI). \\ Pamplona, Norte de Santander, Colombia. \\ E-mail: \{jserrano, pilas\}@unipamplona.edu.co.
}

\begin{abstract}
Resumen: El proceso de desarrollo de software está sujeto a la aplicación de procesos propios de la ingeniería del software (levantamiento de Requerimientos, Análisis, Diseño, Construcción, Pruebas e implementación) que garantizan un flujo correcto de actividades que tienen como objetivo la generación de una herramienta informática que cumpla con las características deseadas por el cliente. La aplicación de esta proceso de la ingeniería del software no garantiza el éxito rotundo del proyecto, pues es de gran importancia validar otros factores que influyen en el desarrollo del mismo y que dependen de la gestión del gerente del proyecto y su equipo como serian tiempos de entrega, alcance del proyecto y por último y en el cual centraremos nuestro estudio la definición de los costos asociados al proyecto pues este factor se considera neurálgico en el éxito o fracaso del proyecto.
\end{abstract}

Palabras clave: Costos, PMBOOK, proyectos, presupuesto.

\begin{abstract}
The software development process is subject to the application of own software engineering processes (Requirements Elicitation, Analysis, Design, Construction, Testing and Implementation) that guarantee a correct activities flow that have like objective generating a Computing tool that comply with the characteristics desired by the customer. But the application of this software engineering process does not guarantee the resounding success of the project, since it is of great importance validating other factors that influence the development of the project and that depend on the management of the project manager and his team, as would be the delivery's times, the project's scope and finally, and in which we will focus our study, the definition of the costs associated with the project because this factor is considered as neuralgic in the success or failure of the project.
\end{abstract}

Keywords: Costs, PMBOOK, Projects, budget.

\section{INTRODUCCIÓN}

Gracias al auge que ha tenido la tecnologías de la información y las comunicaciones en las actividades de la vida cotidiana, y en gran proporción a las actividades empresariales se han abierto las puertas para que entidades con la capacidad de brindar servicios tecnológicos surjan y se posesionen en el mercado; este es el caso del Centro de Investigación Aplicada y Desarrollo en Tecnologías de Información (CIADTI) adscrito a la Universidad de Pamplona el cual en sus 15 años de 
servicio a la comunidad ha desarrollado la suite Academusoft que es una herramientas de Planificación de Recursos Empresariales (ERP por su definición en inglés como Enterprise Resource Planning) orientada a apoyar los procesos académicos y administrativos de una Institución de Educación Superior. Surge una gran incógnita que no solo afecta al CIADTI si no ha todas las empresas del sector de desarrollo de software ¿Cuánto se debe cobrar por los servicios prestados? Para poder dar respuesta a este cuestionamiento se debe definir el alcance de la comercialización del software; es decir, construir software o nuestro compromiso es la de brindar un servicio que permita la generación de una herramienta informática basado en la necesidad del cliente y el cual finalizará cuando el producto haya sido aceptado por el mismo y entre en funcionamiento.

\section{ENTENDIENDO LOS COSTOS}

Este es uno de los factores primordiales al momento de validar si el proceso productivo de una empresa genera beneficios pérdida, pues si conociendo cuánto cuesta la generación de un producto o servicio se podrá determinar de forma acertada el precio de venta del mismo al público.

Los costos se expresan como el valor monetario que se deben utilizar en el ejercicio normal de la actividad económica destinada a la generación de un bien o servicio, pues todo proceso de producción tiene involucrado el consumo o desgaste de una serie de componentes que intervienen en la actividad.

Contar con un proceso de gestión de costos bien definido brinda una herramienta valiosa en la toma de decisiones pues si conocemos de antemano cuánto vale realizar una actividad se podría definir si un proyecto es rentable o no; también tiene gran importancia en las organizaciones contar con un proceso de costo bien definido pues brinda las bases para la búsqueda de productos o servicios de calidad gastando la menor cantidad de recursos, logrando así reducir precio de venta al usuario final y utilizar de forma eficiente y eficaz los recursos de la organización.

En muchas oportunidades los gerentes, directores de oficina y juntas directivas tienen que enfrentar situaciones en las cuales en necesario la toma de decisiones que afectan a la empresa y contar con información de los costos y gastos de la organización son de gran importancia para la toma de decisiones de forma acertada y a tiempo.

\subsection{Costos Directos}

Los costos directos son todos aquellos flujos de recursos que se incorporan físicamente en el desarrollo del producto o servicio, y todos los recursos para la elaboración y/o transformación de los recursos, cada uno de estos costos hacen parte del producto o servicio final.

Entre los rubros más relevantes de estos podemos encontrar (Valenzuela, 2017)

a. Materias Primas: Son los Recursos Materiales que serán transformados en el proceso productivo y se incorporan en el producto o servicio final. Como podría ser la harina con la cual se fabrica el pan o la arena con la cual se elabora un ladrillo.

b. Materiales Directos: Son recursos transformados que no son parte del producto final pero que son necesarios para el proceso de comercialización y sin estos no se lograría la entrega del mismo a los usuarios finales como podrían ser los envases, envolturas etiquetas entre otros.

c. Mano de Obra Directa: Son los recursos de personal necesaria para la creación del producto o servicio y los cuales se encargan de tomar la materia prima y convertirla en el producto final, en el análisis de estos se deben tener en cuenta los salarios, beneficios a trabajadores, aportes a seguridad social, primas horas extras entre otros, como ejemplo de estos tenemos los obreros que operan la máquina que crea los bloques y si hablamos de servicios como producir software hablamos de los ingenieros desarrolladores.

\subsection{Costos Indirectos}

Son todos aquellos costos asociados a recursos que intervienen en el proceso productivo del bien o servicio y que no se incorporan físicamente el bien final. Estos costos hacen parte del proceso de producción. En la actualidad con la automatización de los procesos estos costos han adquirido gran relevancia.

Entre los rubros más representativos de los costos indirectos están (Valenzuela, 2017):

a. Materiales Indirectos: Este los constituyen los Insumos que no tiene partición en el producto elaborado ni forman parte del mismo, pero que son necesarios para la producción del bien o servicio entre los más comunes para estos 
podríamos encontrar herramientas, equipo de trabajo, útiles de aseo entre otros.

b. Mano de Obra Indirecta: En este rubro se tiene en cuenta la mano de obra que no participa en el proceso productivo que genera el producto o servicio, como ejemplo de estos podemos encontrar al gerente de departamento, jefes de áreas, secretarias, etc., es importante tener en cuenta que se debe incluir salarios, beneficios a trabajadores, aportes a seguridad social, primas horas extras entre otros.

c. Gastos de Administración: Comprenden la relación de gastos en los que incurre la organización para el funcionamiento administrativo y no hacen parte del proceso de producción del bien o servicio, en este rubro podemos encontrar al gerente general, y el personal de las áreas administrativa como Contabilidad, Talento Humano, Contratación etc., y debe incluir salarios, beneficios a trabajadores, aportes a seguridad social, primas horas extras entre otros. También hacen parte de este los gastos como energía eléctrica, agua, telefonía, seguros de bienes, elementos de oficina que apoyan el proceso administrativo de la organización.

d. Depreciación: Este es un costo que se establece para cada uno de los bienes que participan activamente en el proceso de productivo y se basa en aplicar una deducción por el desgaste que sufre los activos fijos durante su vida útil generado por el uso que se haga de él.

e. Costo Financiero: hacen referencia a los gastos en los que incurre la organización pro hacer uso del sistema financiero, entre estos podemos destacar los costos financieros aplicados a las cuentas bancarias de la organización y los intereses generado por un crédito en el cual incurre la organización para lograr financiar el proceso productivo

\section{EVOLUCIÓN HISTÓRICA DE LOS COSTOS}

El control de los costos asociados a los procesos productivos de la sociedad se podría remontar a las primeras civilizaciones en procesos como la de producción de bienes de consumo y el comercio, basando su control a los flujos de gasto en dinero realizados por las personas, este procedimiento era un control de los recursos necesarios para la producción de un bien o la prestación de un servicio lo que podríamos asimilar a los actuales manuales de costos, sistema utilizado en algunas industrias como la producción de viñedos, impresión de libros y acerías (Capers Jones, 1998 pp. 9).

El origen formal de la contabilidad de costos se remonta los años 1485 y 1509, antes de la revolución industrial años en los cuales los procesos de manufactura se basan en la transformación de materias primas a través de talleres de artesanos los cuales además de este proceso se encargaban de la comercialización y sumado a esto eran Mono-Productoras, con este proceso simple de producción la información de costoo era muy sencilla se basaba en costos de materia prima y mano de obra directa, el total de estos ítems era aplicado a la cantidad de producto elaborado en un periodo de tiempo.

Con el auge que genera la revolución industrial a mediados de 1776 en las empresas que pasan de los procesos artesanales a procesos industrializados y grandes fábricas que se convierten en poliproductoras, que por su complejidad crean la necesidad de desarrollar un nuevo modelo para la contabilidad de costos y es así como en 1778 se empiezan a llevar formalmente libros auxiliares que registraban todos los elementos que tuviesen influencia en el desarrollo del producto (materia prima, salarios, material de trabajo entre otros).

Entre los años 1890 y 1975 la contabilidad de costos logra su consolidación pues se define su estructura dentro de las cuentas generales esto en potencias mundiales como estados Unidos e Inglaterra y se introduce el concepto de distribución de costos indirectos en procesos de producción, se convierte el proceso de costos como parte de la información financiera de las entidades.

Con la gran depresión, que no es más que la crisis económica mundial que se presentó en los años 30 la contabilidad toma el rol de herramienta de planeación y control, lo cual genero la necesidad de crear métodos que permitieran anticiparse y nacen los conceptos de costos predeterminados (se calculan antes de hacerse o de terminarse el producto) y costos estándar (Representan la cantidad que según la empresa, costará realmente un producto o la operación de un proceso, durante cierto período.) que serían de gran importancia en la dirección de las organizaciones.

En los años 40 y 50 con la demanda incesante de producir bienes y servicios para la pos-guerra el proceso de costoo se centra en definir los costos de producción y materia prima, y se realiza la 
definición de costo de mano de obra basado en tiempos de utilización de este recurso, y surge la necesidad de aplicar costos indirectos a cada uno de los productos elaborados por la empresa, para mediados de los años 85 nace la necesidad de aplicar un nuevo método de costoo basado en actividades (que buscan evaluar el costo de cada una de las tareas que se realizan para la creación de un producto y busca reducir las tareas que no generan) y que hoy día se conoce como costos $\mathrm{ABC}$ y que es el modelo tradicional aplicado en las empresa en la actualidad.(Ricardo A. Billene, 1999).

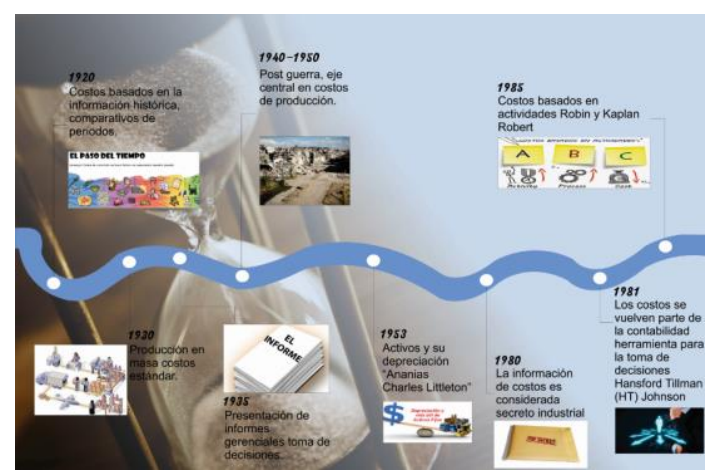

Fig. 1. Evolución Costos Siglo XX Fuente: Ricardo A. Billene, 1999

\section{LOS COSTOS VISTOS DESDE PMBOK}

Este define un marco de estructura formal para desarrollar proyectos, orientando a los gerentes en los pasos a seguir en el desarrollo del mismo y como obtener los resultados esperados, por ende se entiende que cada proyecto tendrá que definir sus particularidades y adaptar el contenido de la guía a su necesidad sin ser esta última una camisa de fuerza que se debe seguir al pie de la letra. Y enmarca dentro del cuerpo del conocimiento un área dedicada a costos en la cual se Incluye los procesos involucrados en estimar, presupuestar y controlar los costos de modo que se complete el proyecto dentro del presupuesto aprobado, basa su definición principalmente en el conocimiento del alcance que tendrá el proyecto, pues es necesario conocer los requisitos de los interesados para determinar acertadamente el presupuesto del proyecto. Y plantea cuatro etapas para el desarrollo del proceso de la Gestión de los Costos del Proyecto

a. Planificar la Gestión de los Costos: Es el proceso que establece las políticas, los procedimientos y la documentación necesarios para planificar, gestionar, ejecutar el gasto y controlar los costos del proyecto.

b. Estimar los Costos: Es el proceso que consiste en desarrollar una aproximación de los recursos financieros necesarios para completar las actividades del proyecto.

c. Determinar el Presupuesto: Es el proceso que consiste en sumar los costos estimados de las actividades individuales o de los paquetes de trabajo para establecer una línea base de costo autorizada.

d. Controlar los Costos: Es el proceso de monitorear el estado del proyecto para actualizar los costos del mismo y gestionar posibles cambios a la línea base de costos.

\section{TÉCNICAS PARA EVALUAR COSTOS EN PROYECTOS}

Gestión del Valor Ganado/Earned Value Management (EVM), técnica que permite medir el desempeño del cronograma y los costos del proyecto a través de la validación del trabajo que se está completamente realizado en un tiempo determinado como lo de define el PMBOK es "Una metodología de gestión para integrar alcance, cronograma y recursos, y para medir el rendimiento y el avance del proyecto en forma objetiva. El rendimiento se mide determinando el costo presupuestado del trabajo realizado y comparándolo con el costo real del trabajo (es decir, el costo real) ("Consultoría y entrenamiento en procesos, herramientas y técnicas relacionadas con la gerencia de proyectos (Project Management, 2012)

Para lograr un análisis efectivo del valor ganado es necesario evaluar tres aspectos relevantes en el instante de su definición ¿Cuál fue el trabajo planificado?, ¿Cuánto trabajo se ha completado? y ¿cuál es el costo en el que se ha incurrido?

La respuesta al primer interrogante nos muestra el valor planeado (PV) de las tareas proyectadas a realizar en el intervalo de tiempo analizado, el segundo cuestionamiento nos revela el valor ganado (EV) que representa el costo presupuestado para el desarrollo de las tareas completadas al momento del análisis, y por último el costo real del trabajo realizado (AC)("Gestión del valor ganado," 2017). 
Teniendo estas variables podemos validar factores que nos permiten conocer cómo avanza el proyecto con relación a los costos estimados entre los factores a analizar encontramos: Índice de Desempeño del Proyecto se puede obtener con el Valor Ganado entre el Valor Planificado, también se podría medir el Índice de Desempeño del Costo el cual se obtiene de dividir el Valor Ganado entre Costo Real y la Variación del Costo el cual se obtiene de la diferencia entre el Valor Ganado y el Costo Real.

\section{ESTADÍSTICAS EN PROYECTOS DE SOFTWARE STANDISH GROUP}

Con el auge de las tecnologías de la Información y las comunicaciones a nivel mundial es importante conocer la efectividad de los proyectos desarrollados en esta área y cuáles son los factores predominantes que evidencian el éxito o el fracaso de un proyecto; para tener un panorama de este tipo de estudios centraremos la mirada en el informe del caos del año 2015, publicado por Standish Group dese 1994.

A continuación se visualiza el reportes del año 2015 y un comparativo con años anteriores es importante resaltar que para la entrega de información del año en mención se analizaron alrededor de 50.000 proyectos de software que van desde pequeños proyecto hasta grandes procesos de sistematización y reingeniería, para el estudio realizado el factor de éxito lo componen el cumplimiento del plazo, del presupuesto y satisfacción del cliente que reemplaza la variable de alcance de la triple restricción.(Group, 2017) .

\section{Análisis de los Proyectos y su Éxito en el 2015}

A través del siguiente gráfico podemos visualizar la información recolectada dl desarrollo de los proyectos y su comportamiento según la escala de exitosos (Cumplieron al $100 \%$ con los factores evaluados), Cambiados (alguna de las variables evaluada fue medicada para poder cumplir con el proyecto) y Cancelados (Cancelación del proyecto).

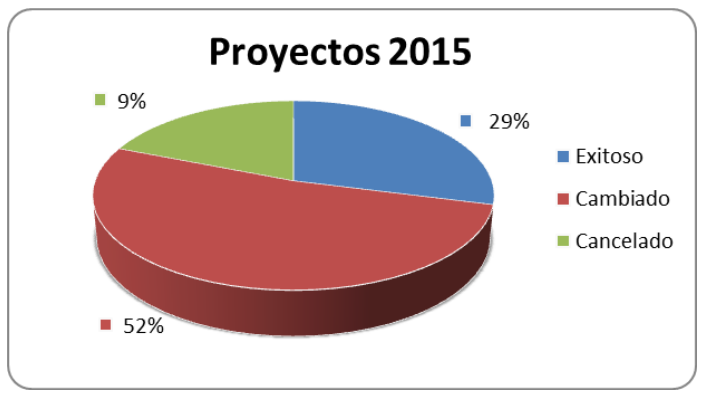

Fig. 2. Evaluación Proyectos 2015. Fuente: Standish Group

Como una forma de ver la evolución de los proyectos de software en los últimos años se visualizará la información obtenida por Standish Group en los últimos cinco años (Group, 2017).

\section{EL PRESUPUESTO COMO FACTOR CLAVE}

Este es un factor muy relevante a tener en cuenta en la validación de éxito o fracaso en los proyectos a continuación se describe los sobrecostos que experimentan las empresas para lograr el éxito de los proyectos dependiendo del tamaño de la misma tomado (Group, 2017).

Tabla 1: Sobrecostos Proyectos

\begin{tabular}{|c|c|}
\hline Tamaño & \% SOBRECOSTOS \\
\hline Pequeña & $214 \%$ \\
\hline Medianas & $182 \%$ \\
\hline Grandes & $178 \%$ \\
\hline \multicolumn{2}{|c|}{ Fuente: Standish Group }
\end{tabular}

En promedio para 2015 los sobrecostos en general para los proyectos se ubicaron en $189 \%$ por encima del valor establecido en su proceso de definición inicial.

Como se puede observar en los resultados obtenidos el proceso de obtención de costos en los proyectos informáticos es ineficiente y es necesario definir estrategias que permitan a las empresas definir de forma exacta cuánto cuesta el desarrollo de los proyectos y qué factores son los más relevantes a identificar y costear.

\section{CONCLUSIONES}

Podemos evidenciar que la gestión de costos no es una área en la cual no se esté improvisando pues ha venido evolucionando en los últimos siglos recopilando conocimiento que tiene aplicación en 
todas los sectores de la economía mundial generando un gran valor para las áreas estratégicas del negocio brindado información relevante para la toma de decisiones y brindando apoyo fundamental en el éxito de los proyectos.

Pero hemos evidenciado en los estudios referenciados en este artículo que los procesos de desarrollo de software no han logrado afinar el proceso de costos lo cual ha generado que un gran porcentaje de estos no logren desarrollarse con las condiciones iniciales y generando afectación en alguna de las aristas primordiales de un proyecto como son de tiempo, alcance y presupuesto.

La construcción de software es una ciencia que se está hasta ahora incubando y necesita que se le hagan revisiones constantes que permitan afianzar el conocimiento de la misma, sumado a este inconveniente lo proceso a los cuales se les aplica el proceso de construcción son variables y sujetos a la imaginación del cliente.

\section{REFERENCIAS}

Capers Jones. (1998). Estimación de costos y administración de proyectos de software (2 a ed.)-capers jones-9789701067055 Añadir Estimación de costos y administración de proyectos de software. (MacGraw Hill, 2 Ed.)

Mauricio, J., \& Pico, M. (2014). Evaluación de la gestión de costos.

PMI. (2013). Guía de los fundamentos para la dirección de proyectos (guía del $\mathrm{PMBOK}^{\circledR}$ ). Project Management Institute, Inc.

Porter, M. (1985). Cadena de Valor. Pirámide.

Ricardo A. Billene. (1999). Análisis de costos I. (E. J. Yuno, Ed.).

Roberto Hernández Sampieri. (2010). Metodología de la Investigación.

Caballero Amaury, Velasco Gabriel, Pardo García A. (2013). Differentiations of objects in diffuse databases. Revista Colombiana de Tecnologías de Avanzada. 2 (22). Pág. 131 - 137.

Pamplona, S. I. de G. U. de. (2013). pac_01_control_documents.

Durán Acevedo Christian M, Iturriago Ali Xavier. (2012). Automatización de un Sistema de Suministro de Agua Potable a Través de la Tecnología Zigbee. Revista colombiana de tecnologías de Avanzada 1 (19), Pág. 36 - 42.

Rodrigo Alvear Tristancho, Laura Patricia Villamizar Carrillo. (2016-II). El Antes y Ahora de la Implantación de Aplicaciones
Informáticas. . Revista colombiana de tecnologías de Avanzada.

Liliana Fernández Samacá, Luís Ariel Mesa Mesa, Wilson Javier Pérez Holguín. (2016-II). Investigación Formativa para Estudiantes de Ingeniería Utilizando Robótica. Revista Colombiana de Tecnologías de Avanzada.

\section{SITIOS WEB}

Consultoría y Entrenamiento en procesos, herramientas y técnicas relacionadas con la gerencia de proyectos (Project Management). (2012). Retrieved February 10, 2017, from http://proyectics.blogspot.com.co/2008/09/valor -ganado-frmulas.html

Diccionario de la Real Academia de la Lengua Española. (2017). Retrieved January 15, 2017 , from http://dle.rae.es

Gestión del valor ganado. (2017). Retrieved February 6, 2017, from https://americalatina.pmi.org/latam/Knowledge Center/Articles/ /media/2B437B5C09974800A 9EE8654AE0323C0.ashx

Group, S. (2017). Chaos Report. Retrieved February 1, 2017, from https://www.standishgroup.com/

Pamplona, C. U. de. (2016). Centro de Investigación Aplicada y Desarrollo en Tecnologías de Información. Retrieved October 18, 2016, from http://plataforma.unipamplona.edu.co/

Soporte Tecnológico Universidad de Pamplona. (2016). Clientes. Retrieved January 21, 2017, from

http://www.unipamplona.edu.co/unipamplona/p ortalIG/home_38/recursos/01_general/2806201 $1 /$ clientes.jsp

Valenzuela, E. (2017). GESTION DE COSTOS. Retrieved January 22, 2017, from http://gerenciadeproyectos88.blogspot.cgggom. co/2008/08/gestion-de-costos.html 